INVITED PAPER Special Section on Leading-Edge Applications and Fundamentals of Superconducting Sensors and Detectors

\title{
Superconducting On-Chip Spectrometery for Millimeter-submillimeter Wave Astronomy
}

Akira ENDO $^{* * a)}$, Nonmember

SUMMARY Since the birth of astrophysics, astronomers have been using free-space optics to analyze light falling on Earth. In the future however, thanks to the advances in photonics and nanoscience/nanotechnology, much of the manipulation of light might be carried out using not optics but confined waveguides, or circuits, on a chip. This new generation of instruments will be not only extremely compact, but also powerful in performance because the integration enables a greater degree of multiplexing. The benefit is especially profound for space- or air-borne observatories, where size, weight, and mechanical reliability are of top priority. Recently, several groups around the world are trying to integrate ultra-wideband (UWB), low-resolution spectrometers for millimeter-submillimeter waves onto microchips, using superconducting microelectronics. The scope of this Paper is to provide a general introduction and a review of the state-ofthe-art of this rapidly advancing field.

key words: Spectroscopy, millimeter wave, submillimeter wave, superconducting electronics, astronomical instrumentation

\section{Introduction: Emergence of Superconducting On- chip Spectrometers for mm-submm Wave Astron- omy}

Ultra-wideband $\left(\mathrm{UWB}^{* *}\right)$, low-resolution $(R \sim 100-1000)^{* * *}$, direct-detection spectrometers for millimeter-submillimeter $(\mathrm{mm}$-submm) waves $(100-1000 \mathrm{GHz})$ have recently identified several unique applications in the field of astronomy [1]: for example, rapid redshift determination of very distant galaxies [2], and diagnostic surveys of emission/absorption lines from interstellar media [3]. At the same time, advances in mm-submm wave detector technology for constructing large arrays of detectors with high sensitivity have made the realization of such spectrometers feasible. The first generation of such spectrometers, which are currently in operation, are based on optical gratings or optical fourier transform spectrometers, much like those that have been used in the

Manuscript received July 7, 2014.

Manuscript revised October 1, 2014.

†The author is with the Department of Electrical Engineering, Faculty of Mathematics and Computer Science (EEMCS), Delft University of Technology, 2628 CD Delft, The Netherlands.

${ }^{*}$ The author is also with the Kavli Institute of NanoScience, Faculty of Applied Sciences, Delft University of Technology, Lorentzweg 1, 2628 CJ Delft, The Netherlands.

Website: http://www.cosmonanoscience.tudelft.nl/people/endo/

a)E-mail: a.endo@tudelft.nl

DOI: 10.1587/transele.E98.C.219

${ }^{* *}$ In this Paper, we loosely define the term ultra-wideband, or UWB, as a relative bandwidth of more than $100 \%$. This is equivalent to saying that the bandwidth is more than 1 octave.

${ }^{* * *} R$ is the resolving power, defined as $R=f_{0} / \Delta f$, where $f_{0}$ and $\Delta f$ are the center frequency the width of one spectral channel, respectively. visible-infrared wavelength domain [1]. However, the next generation of UWB spectrometers for mm-submm wave astronomy could look very different from their optical analogues, because there are currently several attempts to integrate the entire spectrometer on chip, by using the recent advances in superconducting electronics. The aim of this Paper is to provide an introduction and overview towards this emerging field of superconducting on-chip spectrometry for $\mathrm{mm}$-submm wave astronomy.

Historically, the mm-submm wavelength range was first cultivated by extending the technology used in radio astronomy, which is coherent reception of light in the the form of waves, rather than particles [4]. This technique provides very high spectral resolution $\left(R>10^{6}\right)$, and also very high spatial resolution through aperture synthesis interferometry. However, so far it has been difficult to cover an instantaneous bandwidth much greater than $10 \mathrm{GHz}$ using coherent receivers. Moreover, coherent reception of light is fundamentally limited in sensitivity because of quantum noise. Quantum noise becomes an increasingly serious problem at shorter wavelengths, because the photon occupation number of the radiation from astronomical sources drop [5].

In contrast to radio astronomy, in optical astronomy direct-detection spectrometers are widely used. These spectrometers typically have an array of direct detectors (e.g., CCD) behind an optical grating, and achieve a bandwidth in excess of an octave. Until recently, such an approach was difficult in the mm-submm wavelength range, because of the lack of a large array of (direct) detectors with high sensitivity. This situation has changed: the number of pixels in modern mm-submm detector arrays used for imaging cameras is currently in the range of 100-10,000 [6]. Arrays of 100-1000 detectors have been made with semiconducting bolometers, and many of the newer arrays with larger pixel counts use superconducting detectors, either transition edge sensors (TESs) or microwave kinetic inductance detectors (MKIDs).

The realization of large detector arrays has made it possible to introduce the optical spectrometer technology to the mm-submm wavelength domain. The first generation of such instruments, Z-spec [7], and ZEUS [8], used semiconducting bolometers. An upgrade of ZEUS, ZEUS2 [9], uses superconducting transition edge sensors. ZEUS and ZEUS-2 use an optical Echelle grating, where Z-spec uses a waveguide-coupled diffraction grating. Aside from grating spectrometers, Fourier Transform Spectrometers are also being used and developed for new instruments. In par- 
ticular, the SPIRE FTS onboard the Herschel satellite has been used to conduct emission line surveys in the submillimeter band from space [10]. The 10,000 pixel SCUBA2 instrument, now in operation, is also equipped with an FTS [11].

The focus of this Paper is on the recent efforts by a number of groups in integrating the functionality of optical spectrometers onto a microchip, in various configurations. In section 2, I will first describe the background to this movement. In section 3, I will introduce the fundamental principles that are common to all types of superconducting on-chip spectrometers. In section 4 , I will provide a review of the attempts to realize on-chip spectrometers. Some prospects for the future are given in section 5 .

\section{Background}

The realization of mm-submm on-chip spectrometers takes advantage of the timely emergence and advances of several superconducting electronic components. It is also very interesting that there is a growing field of "astrophotonics" in the visible-infrared wavelength domain, which has a great deal of similarity in philosophy and methodology with the development of on-chip spectrometers in the mm-submm. In this section, we will touch upon these subjects, to appreciate the soil from which on-chip spectrometers have sprouted out.

\subsection{Maturation of the Enabling Technologies}

\subsubsection{MKIDs}

MKIDs were invented little before 2003 [12], as a detector that will meet the demands for constructing arrays of $\gg 1000$ pixels with high sensitivity. Since then, MKIDs have shown photon-noise limited sensitivity down to Noise Equivalent Powers below of $10^{-18} \mathrm{~W} \mathrm{~Hz}^{-0.5}$ [13] in the mm-submm domain, and a camera with $>20,000$ MKID pixels is under preparation. There are several detailed review papers that focus on MKIDs [14]-[16].

MKIDs are superconducting microwave resonators that are made sensitive to external radiation. When a photon is absorbed in an MKID, it initially breaks a Cooper pair to create two quasiparticles, which can further excite more quasiparticles depending on their energy [17]. This excitation causes both the surface inductance and the surface resistance of the superconducting film to increase. As a result, the resonance frequency is shifted downwards and the resonant $\mathrm{Q}$ factor is reduced. By monitoring either of these variables, the MKID is used as a detector. Crucially, by changing the microwave resonance frequency of each MKID, many MKIDs can be multiplexed in frequency domain on one transmission line, thereby allowing simultaneous readout of $>1,000$ elements with only a single pair of coaxial cables having to reach the MKIDs at low temperature (typically $100-300 \mathrm{mK}$ ).

MKIDs are naturally suited for use in an on-chip spec- trometer. For example, the NbTiN layer of a NbTiN/Al hybrid MKID [18] is ideally suited for making the antenna and the low-loss submm transmission line required for all types of on-chip spectrometers, and it allows simultaneous fabrication of these components with the MKIDs. MKIDs also do not (in principle) require sophisticated micro-machined heat transfer structures typical for bolometers, because MKIDs are quantum detectors. This gives more freedom in design when combining the detector with the frequencyselecting section of the spectrometer.

\subsubsection{Ultra-wideband radiation coupling}

One of the challenges in utilizing on-chip spectrometers is coupling radiation from the telescope optics onto a transmission line on the chip. In order to take full advantage of the broad band capability of direct detection (to be competitive against coherent detection), one would like to achieve close to an octave bandwidth or even more. Traditionally, a spiral antenna in combination with a dielectric lens has been regarded as the prime candidate for such broad band coupling, though the spiral antenna has a linear polarization angle that rotates with frequency. A good alternative is the sinuous antenna [19], which suppresses the rotation of polarization to within $\sim \pm 5$ degrees. Recently, there has been the advent of the superleaky antenna, which combines broadband radiation coupling with polarization stability [20]. Another direction of development is wide-band coupling using feed horns using micromachining technology [21].

\subsubsection{Low-resolution on-chip filters for multichroic imag- ing}

While the concept of making on-chip spectrometers with a resolution of $R>100$ is relatively new, it can be seen as a natural extension of on-chip multichroic cameras with $R \sim$ 10. The pixels on these chips have an antenna connected to superconducting broadband filters that separate the signal into multiple colors and deliver them into different detectors, which can be for example TESs [19] or MKIDs [22].

\subsection{Parallel Movement: Astrophotonics in the Visible- infrared Domain}

It is worth noting that the on-chip movement is also taking place in the visible-infrared (Vis-IR) wavelength domain: this approach is sometimes called "astrophotonics" [23], [24]. The concept is to pursue compactness, simplicity, more functionality and higher performance, by applying the advancing technology in photonics to astronomical instruments. Among them is the on-chip FTS spectrometer SWIFTS [25], and the 2D array waveguide spectrometer [26], which both have now their superconducting analogue for mm-submm spectrometry as we will see later. For a review of astrophotonics, see references [23], [24]. While there is a $\times 1000$ difference in wavelength and also a difference in applicable materials and techniques, the philosophy 
is quite similar, and therefore the on-chip development in the Vis-IR and in the mm-submm could significantly benefit each other.

\section{Basic Principles of On-chip Spectrometery using Su- perconductiviting Electronics}

There are several advantages and disadvantages in integrating a spectrometer on chip, compared to constructing it using optical components. In this section, we will discuss the basic principles that apply to all variants of superconducting on-chip spectrometers for mm-submm waves.

\subsection{Size}

As is elegantly explained by Mansuripur [27] and articulated in the context of mm-submm spectroscopy by Zmuidzinas [28], the size of an optical spectrometer is related to the observed wavelength and spectral resolution. Heisenberg's uncertainty principle states that in order to achieve an energy resolution of $\Delta E=h \Delta f$, one must introduce an uncertainty in the arrival time of each photon that is at least $\Delta t=h / \Delta E=1 / \Delta f$. In the case of a grating spectrometer with an optical path in vacuum, for example, this corresponds to a minimum path length of $c \Delta t=c / \Delta f$. For example, a conventional optical grating spectrometer would require a minimum physical length of $c / 0.3 \mathrm{GHz}=1 \mathrm{~m}$ to achieve a frequency resolution of $0.3 \mathrm{GHz}$ at $300 \mathrm{GHz}$ (resolving power of 1000).

Superconducting electronics provide several ways of reducing the required physical size of a spectrometer. Firstly, when the signal travels along a transmission line with a phase velocity of $v_{\mathrm{p}}$, this will reduce the required physical length by a factor of $v_{\mathrm{p}} / c$. Note that not only the dielectric material that fills the transmission line, but also the kinetic inductance of the superconductor can significantly contribute to reducing the phase velocity. Secondly, once the signal is confined in a transmission line, then it is relatively straight forward to make the line narrow and to fold it tightly. Thirdly, one could introduce resonant structures (filters) in which the signal is reflected $\sim Q$ times, where $Q$ is the quality factor of the resonator. Since this will introduce an uncertainty in the time that the photon spends in the resonator, equal to the resonator ring time $Q / \pi f$, it will reduce the required physical size by $\sim Q$. While a Fabry-Perot Etalon in optics [27] also benefits from the same reduction in the physical length of the optical path, the filterbank has the added advantage that it is easy to pack many spectral channels with different frequencies very close to each other.

The reduction in size becomes especially crucial in forming an array of spectrometers, to construct an integral field spectrometer. Another case where compactness becomes essential is when the spectrometer should be installed on a space- or air-borne observatory.

\subsection{Alignment Accuracy}

In the case of optical spectrometers, each component must be mechanically aligned with each other, unless they are made out of one metal block. In the case of on-chip spectrometers, the relative position of each element is defined by lithographic techniques, which typically have sub-micron precision.

\subsection{Dynamic Adjustment of the Optical/Electrical Path Length}

Some types of spectrometers rely on the ability to dynamically change the optical path length. In the case of optical spectrometers, this is achieved by actually moving components like mirrors, which adds an extra degree of complexity to the system.

For an on-chip spectrometer, it is difficult to implement a scheme which makes components physically move with respect to each other. Instead, one could use the currentdependence of the superconducting kinetic inductance. This property has been recently exploited in attempt to realize a variable phase shifter [29] and a parametric amplifier [30].

\subsection{Transmission Loss}

One significant disadvantage of using superconducting electronics instead of free-space optics is transmission loss. In principle, optical spectrometers are free of transmission loss as long as the light travels through vacuum (though there is reflection loss at the mirror, and spectrometers that rely on dielectric material are subject to absorption losses as well.) In contrast, on-chip spectrometers using superconducting electronics can suffer from dissipation loss and radiation loss of the transmission lines.

It is perhaps important to realize that, as long as the losses along a continuous transmission line is considered, the amount of loss along the transmission line is independent of whether the spectrometer is resonant (e.g., filterbank: subsection 4.1) or non-resonant (e.g., Mach-Zehnder demultiplexer: subsection 4.4), because in both cases the light must spend an equal average time of $\Delta t$ as explained in subsection 3.1. However, radiation losses are usually dominated by discontinuities, such as the terminations of a resonator, so resonant circuits have a potential disadvantage of suffering from more radiation losses compared to nonresonant circuits.

Regarding dissipation losses, there has recently been a great deal of effort in reducing losses at microwave (1$10 \mathrm{GHz}$ ) frequencies, in terms of both material [31] and geometry [32]. A similar approach in the mm-submm band is probably required in order to realize on-chip spectrometers with high spectral resolution.

The arrayed waveguide grating (AWG) spectrometer (subsection 4.3) is a unique approach, in which the spectral dispersion happens in a 2D quasi free space, which can 
in principle have much less losses compared to transmission lines.

\section{Realizations of Superconducting On-chip Spectrom- eters}

There have recently been a variety of proposals and experimental attempts to make a mm-submm wave on-chip spectrometer using superconducting electronics. Naturally, each of them have a counterpart analogous in operation principle, either in optics or in radio technology. There are also some configurations for optical spectrometers that have not been explored in the mm-submm. In the following, I provide a brief review of each approach.

\section{$4.1 \quad$ Filterbank}

The filterbank is a type of spectrometer that has been commonly used for microwaves and lower frequencies [33]. In Fig. 1, I present a schematic diagram of an on-chip filterbank spectrometer for mm-submm waves. The signal enters the circuit from a broad band antenna (1). Then the signal is carried along a transmission line (e.g., microstrip line, coplanar waveguide) (2). From this signal line, multiple frequency channels branch out. Each channel consists of a narrow band pass filter (3) and a detector (4). The signal that is not absorbed in any of the channels gets absorbed at the end of the signal line, typically with a lossy piece of transmission line (5).

There are currently two groups that have reported experimental results on superconducting on-chip filterbank spectrometers. One is the SuperSpec group, which has reported a resolution of $R=300-700$ in the $195-310 \mathrm{GHz}$ band [34]. The other is the DESHIMA group, which has reported a dissipation-limited internal resonator $Q$ of 140-350 in the $600-700 \mathrm{GHz}$ band [35]. Both groups have used distributed microstrip line resonators for the $\mathrm{mm}$-submm wave filters, and MKIDs for the detectors.

As explained in subsection 3.1, the filterbank spectrometer folds the required electrical path length within the resonant filter, by a factor of $Q$. This property can be used to make the system compact. On the other hand, it puts a strong demand on the lithographic process that determines the length of the resonant filters [36].

\subsection{SWIFTS}

The SWIFTS (Stationary-Wave Integrated Fourier-Transform Spectrometry) is a Fourier transform spectrometer integrated on a chip. In this configuration, an array of detectors is used to sample the shape of the standing wave that is created by the incoming signal. Figure 2 is a schematic diagram of a superconducting SWIFTS spectrometer. The signal enters the circuit from a broad band antenna (1), and is fed to a low-loss transmission line (2). The transmission line is terminated with an open end, where the signal is reflected back towards the antenna. This creates a standing

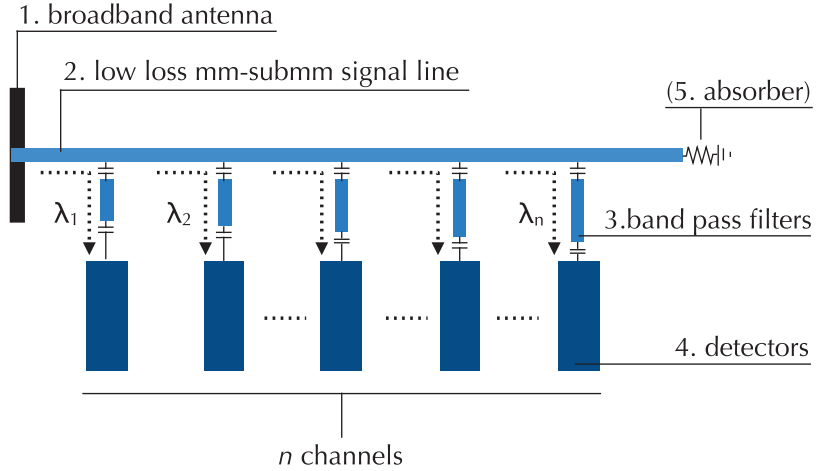

Fig. 1 Schematic diagram of an on-chip filterbank spectrometer for mmsubmm waves. The signal enters the circuit from a broad band antenna (1). Then the signal is carried along a transmission line (e.g., microstrip line, coplanar waveguide) (2). From this signal line, multiple frequency channels branch out. Each channel consists of a narrow band pass filter (3) and a detector (4). The signal that is not absorbed in any of the channels gets absorbed at the end of the signal line, typically with a lossy piece of transmission line (5).

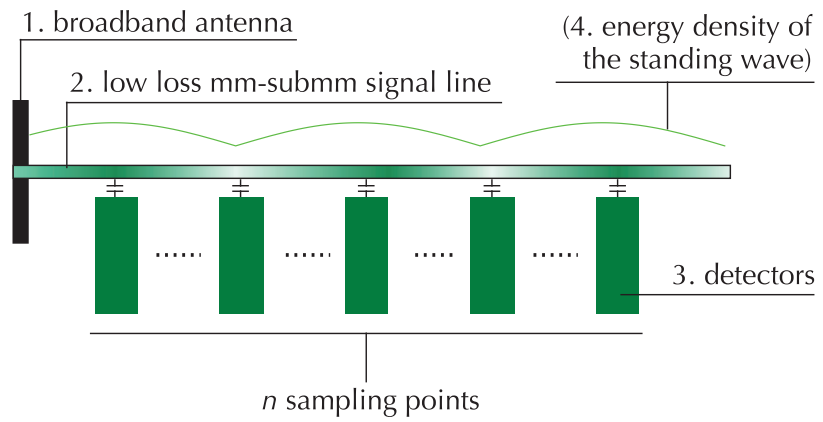

Fig. 2 Schematic diagram of a superconducting SWIFTS spectrometer [38] for mm-submm waves. The signal enters the circuit from a broad band antenna (1), and is fed to a low-loss transmission line (2). The transmission line is terminated with an open end, where the signal is reflected back towards the antenna. This creates a standing wave along the line (4). Along the signal line, there is an array of detectors (3), each weakly coupled to the signal line, absorb part of the signal and thereby probe the shape of the standing wave.

wave along the line (4). Along the signal line, there is an array of detectors (3), each weakly coupled to the signal line, absorb part of the signal and thereby probe the shape of the standing wave. The Fourier transform of the waveform provides the spectrum of the original signal. Despite the discrete sampling of the waveform, SWIFTS can theoretically reach an optimum optical efficiency of $74 \%$ as shown by le Coarer et al. [25].

Following the demonstration in the optical [25] and RF domain [37], Boudou et al. have conducted initial experiments of a mm-wave version [38], using MKIDs for the detectors. As the authors state, the SWIFTS spectrometer has a sensitivity penalty at low photon occupation numbers, in the same manner as an optical FTS [39].

\subsection{Waveguide Grating}

An arrayed waveguide grating (AWG) spectrometer replaces the optical grating of a classical optical grating spec- 


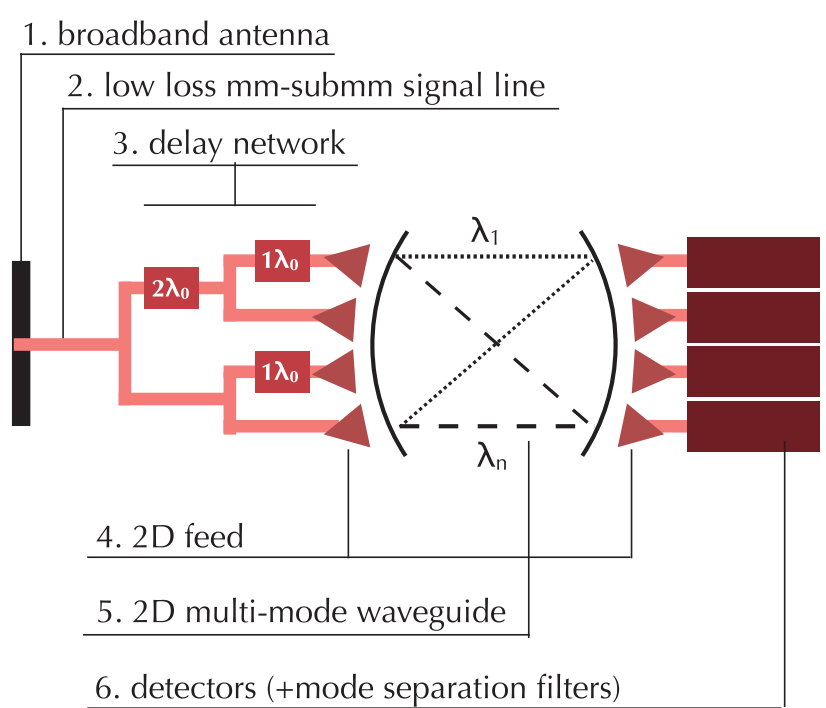

Fig. 3 Schematic diagram of an on-chip array waveguide grating spectrometer [41] for mm-submm waves. The signal enters the chip via a broadband antenna (1), and is fed to a low loss superconducting signal line (2). The signal line branches into a network of transmission lines, which have a different phase delay depending on the path (3). At the output of the delay network, the signal is casted out into a multi-mode $2 \mathrm{D}$ waveguide using 2-D feed horns. The signal is sampled at the opposite side of the waveguide. The interference of the signal separates each wavelength into different output channels.

trometer with an array of feeds. The input signal is separated into multiple waveguides, which connect to the interfering section with a gradient in phase delay. AWG spectrometers have been commercialized in the visible domain for telecommunications, and are considered to be used for future large scale instruments for optical astronomy [40]. A mm-submm version is being developed by the $\mu$-Spec group [41]. A conceptual drawing of a mm-submm waveguide grating spectrometer, much like the one developed by Cataldo. et al. [41], is presented in Fig. 3. The signal enters the chip via a broadband antenna (1), and is fed to a low loss superconducting signal line (2). The signal line branches into a network of transmission lines, which have a different phase delay depending on the path (3). At the output of the delay network, the signal is casted out into a multi-mode 2$\mathrm{D}$ waveguide using 2-D feed horns. The signal is sampled at the opposite side of the waveguide. The interference of the signal separates each wavelength into different output channels. When the signal is broadband, then the signal at the output will contain multiple frequency signals corresponding to different grating orders. In that case, the multiple orders can be further separated by using a low resolution filterbank [41].

\subsection{Cascaded Mach-Zehnder Demultiplexer}

The cascaded Mach-Zehnder (CMZ) demultiplexer has been proposed and demonstrated on chip in the field of photonics at visible wavelength [42]. It is straight forward to think of a mm-submm analogue, as shown in Fig. 4. The signal enters from a broadband antenna (1), and is fed into a low-

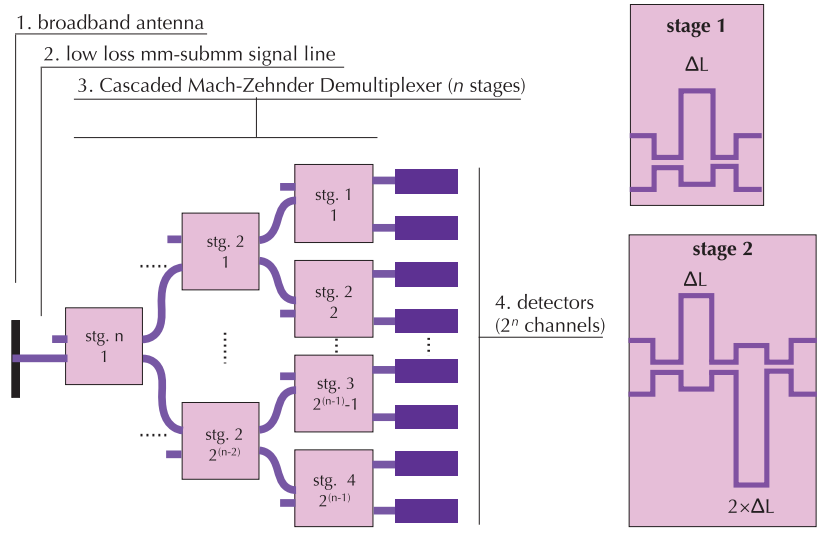

Fig. 4 Schematic diagram of an on-chip cascaded Mach-Zehnder spectrometer [42] for mm-submm waves. The signal enters from a broadband antenna (1), and is fed into a low-loss transmission line (2). Then the signal is separated into different frequencies in a multi-stage Mach-Zehnder spectrometer. The signal is first split into two, one containing the even frequency channels and the other containing the odd frequency channels.

loss transmission line (2). Then the signal is separated into different frequencies in a multi-stage Mach-Zehnder spectrometer. The signal is first split into two, one containing the even frequency channels and the other containing the odd frequency channels. This first stage (stage $n$ in Fig. 4) has the highest, hence final, frequency resolution. The function of the following stages is to sort out each of these frequency components. The final stage (stage 1 in Fig. 4) has the lowest resolving power.

Compared to a filterbank spectrometer, the CMZ configuration requires a much longer physical length of transmission line because it is not resonant. The disadvantage is that it requires a larger chip area, though this could still be comparable to or less than the area occupied by the detectors. The advantage is that this method allows a much larger fabrication error regarding the length of the transmission line, as is proven by the experimental demonstration in visible wavelengths [42].

\subsection{Fabry Perot}

Another type of optical spectrometer that has been used in mm-submm waves is the Fabry-Perot etalon [1]. This type of spectrometer uses an optical resonant cavity which acts as a band pass filter. The passband can be sweeped in frequency by changing the separation of the mirrors. Hence the full spectrum of the source can be obtained by doing a scan.

When considering an integrated version of a FabryPerot spectrometer, the obvious problem is how to realize a phase shift that is variable. One possibility is to use the current-dependence of the superconducting kinetic inductance (Jochem Baselmans, private communications). By driving a dc current through a superconducting transmission line filter, one could, in principle, shift the kinetic inductance of a wire and therefore the pass band frequency of the filter, according to [15] $\delta L_{k} / L_{k}=\kappa_{*} J^{2} / J_{*}^{2}$. Here, $\delta L_{k}$ is the shift in inductance from the zero-current value $L_{k}, \kappa_{*}$ is a 


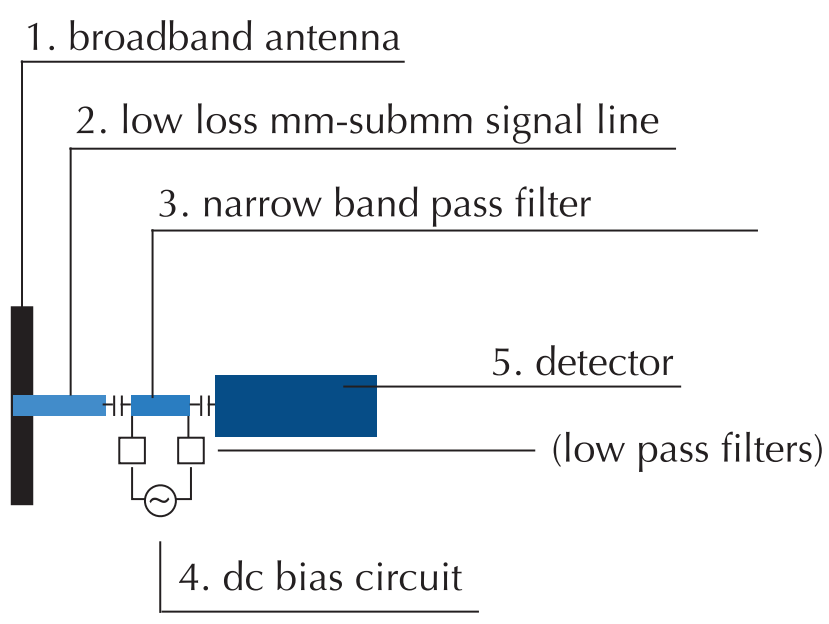

Fig. 5 Schematic drawing of a single-pixel of an on-chip Fabry-Perot spectrometer for mm-submm waves. The signal is first received by a broadband antenna (1), and is fed into a low-loss transmission line (2). Then there is a bandpass filter (3), which determines the frequency component that can reach the detector (4). The passband of the bandpass filter can be controlled electrically by driving a (dc or ac) current through it (5).

dimensionless constant of order unity, $J$ is the current density, and $J_{*}=\sqrt{\pi N_{0} \Delta_{0}^{3} /\left(\hbar \rho_{\mathrm{n}}\right)}$, where $N_{0}$ is the single-spin density of electron states at the Fermi energy, $\Delta_{0}$ is the superconducting gap energy at $T=0$, and $\rho_{n}$ is the normal state resistivity. A schematic drawing of such a spectrometer is presented in Fig. 5. The signal is first received by a broadband antenna (1), and is fed into a low-loss transmission line (2). Then there is a bandpass filter (3), which determines the frequency component that can reach the detector (4). The passband of the bandpass filter can be controlled electrically by driving a (dc or ac) current through it (5).

In the case of an optical Fabry-Perot spectrometer, one can place an array of detectors behind one etalon, so that one can observe multiple positions on the sky during one scan. In the case of the on-chip version, one would need to have one filter per pixel. While the size of the filter itself is small and should not be a problem, the method for applying a bias current would need to be carefully considered so that it does not occupy too much space on the chip and/or interfere with the detector.

\section{Future Prospects}

As we have seen, it is possible to think of a superconducting circuit analogue of many optical spectrometers in the $\mathrm{mm}$-submm domain. Some of the on-chip spectrometer concepts are already under development, as the key technology for next-generation astronomical spectrometers. The ongoing development of "astrophotonics" in the Vis-IR domain [23], [24] suggests that the integration on chip has the potential of providing not only a more compact and scalable system, but new functionalities that are extremely difficult to realize using free-space optics.

What is next? After all, all of the on-chip concepts that have been discussed in this paper are novel but still do not depart significantly from classical electromagnetism as long as one ignores the microscopic nature of superconductivity and treats it as a conductor with a complex surface impedance. In the future, this could change; recently there have been a number of proposal to utilize techniques of quantum optics for astronomical instruments [43], [44]. The precise and flexible control of light that superconducting circuits offer could provide the soil for the quantumoptical generation of astronomical instruments to flourish in the future, in a way similar to the success of circuit quantum electrodynamics [45].

\section{References}

[1] G. J. Stacey, "THz low resolution spectroscopy for astronomy," IEEE Trans. Terahertz Sci. Technol., vol.1, no.1, pp.241-255, Sept. 2011.

[2] R. E. Lupu, K. S. Scott, J. E. Aguirre, I. Aretxaga, R. Auld, E. Barton, A. Beelen, F. Bertoldi, J. J. Bock, D. Bonfield, C. M. Bradford, S. Buttiglione, A. Cava, D. L. Clements, J. Cooke, A. Cooray, H. Dannerbauer, A. Dariush, G. De. Zotti, L. Dunne, S. Dye, S. Eales, D. Frayer, J. Fritz, J. Glenn, D. H. Hughes, E. Ibar, R. J. Ivison, M. J. Jarvis, J. Kamenetzky, S. Kim, G. Lagache, L. Leeuw, S. Maddox, P. R. Maloney, H. Matsuhara, E. J. Murphy, B. J. Naylor, M. Negrello, H. Nguyen, A. Omont, E. Pascale, M. Pohlen, E. Rigby, G. Rodighiero, S. Serjeant, D. Smith, P. Temi, M. Thompson, I. Valtchanov, A. Verma, J. D. Vieira, and J. Zmuidzinas, "Measurements of $\mathrm{CO}$ redshifts with z-spec for lensed submillimeter galaxies discovered in the h-atlas survey," Astrophys. J., vol.757, no.2, pp.135, Oct. 2012.

[3] P. P. van der Werf, K. G. Isaak, R. Meijerink, M. Spaans, A. Rykala, T. Fulton, A. F. Loenen, F. Walter, A. Weiß, L. Armus, J. Fischer, F. P. Israel, A. I. Harris, S. Veilleux, C. Henkel, G. Savini, S. Lord, H. A. Smith, E. González-Alfonso, D. Naylor, S. Aalto, V. Charmandaris, K. M. Dasyra, A. Evans, Y. Gao, T. R. Greve, R. Güsten, C. Kramer, J. Martín-Pintado, J. Mazzarella, P. P. Papadopoulos, D. B. Sanders, L. Spinoglio, G. Stacey, C. Vlahakis, M. C. Wiedner, and E. M. Xilouris, "Black hole accretion and star formation as drivers of gas excitation and chemistry in Markarian 231," Astron. Astrophys., vol.518, no.L42, pp.5, July-Aug. 2010.

[4] J. Zmuidzinas and P. L. Richards, "Superconducting detectors and mixers for millimeter and submillimeter astrophysics," Proc. IEEE, vol.92, no.10, pp.1597-1616, Oct. 2004.

[5] G. B. Taylor, C. L. Carilli, and R. A. Perley, eds., Synthesis Imaging in Radio Astronomy II, Astronomical Society of the Pacific Conference Series, vol.180, 1999.

[6] W. S. Holland, D. Bintley, E. L. Chapin, A. Chrysostomou, G. R. Davis, J. T. Dempsey, W. D. Duncan, M. Fich, P. Friberg, M. Halpern, K. D. Irwin, T. Jenness, B. D. Kelly, M. J. MacIntosh, E. I. Robson, D. Scott, P. A. R. Ade, E. Atad-Ettedgui, D. S. Berry, S. C. Craig, X. Gao, A. G. Gibb, G. C. Hilton, M. I. Hollister, J. B. Kycia, D. W. Lunney, H. McGregor, D. Montgomery, W. Parkes, R. P. J. Tilanus, J. N. Ullom, C. A. Walther, A. J. Walton, A. L. Woodcraft, M. Amiri, D. Atkinson, B. Burger, T. Chuter, I. M. Coulson, W. B. Doriese, C. Dunare, F. Economou, M. D. Niemack, H. A. L. Parsons, C. D. Reintsema, B. Sibthorpe, I. Smail, R. Sudiwala, and H. S. Thomas, "SCUBA-2: The 10000 pixel bolometer camera on the James Clerk Maxwell Telescope," Mon. Not. R. Astron. Soc., vol.430, no.4, pp.2513-2533, Apr. 2013.

[7] C. M. Bradford, P. A. R. Ade, J. E. Aguirre, J. J. Bock, M. Dragovan, L. Duband, L. Earle, J. Glenn, H. Matsuhara, B. J. Naylor, H. T. Nguyen, M. Yun, and J. Zmuidzinas, "Z-Spec: a broadband millimeter-wave grating spectrometer: design, construction, and first cryogenic measurements," Society of Photo-Optical Instrumentation Engineers (SPIE) Conference Series, vol.5498, p.257, Oct. 
2004.

[8] G. J. Stacey, S. Hailey-Dunsheath, T. Nikola, S. C. Parshley, D. J. Benford, S. H. Moseley Jr., J. G. Staguhn, and R. A. Shafer, "Zeus: the redshift (z) and early universe spectrometer," Proc. SPIE, vol.5498, pp.232-245, Oct. 2004.

[9] C. Ferkinhoff, D. Brisbin, T. Nikola, S. Parshley, G. J. Stacey, S. J. Hailey-Dunsheath, K. D. Irwin, H. Cho, M. Niemack, D. J. Benford, J. Staguhn, T. G. Phillips, and E. Falgarone, "Development of the 2nd generation redshift(z) and early universe spectrometer and the detailed study of far-IR fine-structure lines in high-z galaxies," American Astronomical Society Meeting Abstracts, vol.221, pp.414.02, Jan. 2013.

[10] B. M. Swinyard, E. T. Polehampton, R. Hopwood, I. Valtchanov, N. Lu, T. Fulton, D. Benielli, P. Imhof, N. Marchili, J.-P. Baluteau, G. J. Bendo, M. Ferlet, M. J. Griffin, T. L. Lim, G. Makiwa, D. A. Naylor, G. S. Orton, A. Papageorgiou, C. P. Pearson, B. Schulz, S. D. Sidher, L. D. Spencer, M. H. D. Wiel, and R. Wu, "Calibration of the Herschel SPIRE fourier transform spectrometer," Mon. Not. R. Astron. Soc., vol.440, no.4, pp.3658-3674, June 2014.

[11] B. Gom, D. A. Naylor, P. Friberg, and G. Bell, "Commissioning of fts-2, the scuba-2 imaging fourier transform spectrometer," Imaging and Applied Optics, p.JTu4A.4, Optical Society of America, 2013.

[12] P. K. Day, H. G. LeDuc, B. A. Mazin, A. Vayonakis, and J. Zmuidzinas, "A broadband superconducting detector suitable for use in large arrays," Nature, vol.425, no.6960, pp.817-821, Oct. 2003.

[13] P. J. de Visser, J. J. A. Baselmans, J. Bueno, N. Llombart, and T. M. Klapwijk, "Fluctuations in the electron system of a superconductor exposed to a photon flux," Nat. Commun., vol.5, Feb. 2014.

[14] B. A. Mazin, "Microwave kinetic inductance detectors: The first decade," in American Institute of Physics Conference Series, ed. B. Young, B. Cabrera, and A. Miller, American Institute of Physics Conference Series, vol.1185, pp.135-142, Dec. 2009.

[15] J. Zmuidzinas, "Superconducting microresonators: physics and applications," Annual Review of Condensed Matter Physics, vol.3, pp.169-214, 2012.

[16] J. Baselmans, "Kinetic inductance detectors," J. Low Temp. Phys., vol.167, no.3-4, pp.292-304, May 2012.

[17] M. Kurakado; "Possibility of high-resolution detectors using superconducting tunnel-junctions," Nucl. Instrum. Methods Phys. Res., vol.196, no.1, pp.275-277, May 1982.

[18] R. M. J. Janssen, J. J. A. Baselmans, A. Endo, L. Ferrari, S. J. C. Yates, A. M. Baryshev, and T. M. Klapwijk, "High optical efficiency and photon noise limited sensitivity of microwave kinetic inductance detectors using phase readout," Appl. Phys. Lett., vol.103, no.20, pp. 203503, Nov. 2013

[19] A. Suzuki, K. Arnold, J. Edwards, G. Engargiola, A. Ghribi, W. Holzapfel, A. T. Lee, X. F. Meng, M. J. Myers, R. O'Brient, E. Quealy, G. Rebeiz, P. Richards, D. Rosen, and P. Siritanasak, "Multichroic dual-polarization bolometric detectors for studies of the cosmic microwave background," Society of Photo-Optical Instrumentation Engineers (SPIE) Conference Series, vol.8452, Sept. 2012.

[20] A. Neto, N. Llombart, I. Lager, J. Baselmans, A. Baryshev, and S. Yates, "Leaky lens antennas and kinetic inductance detectorsthe solution for THz integration," European Microwave Conference (EuMC), 2013, pp.354-356, Oct. 2013.

[21] J. W. Britton, J. P. Nibarger, K. W. Yoon, J. A. Beall, D. Becker, H. M. Cho, G. C. Hilton, J. Hubmayr, M. D. Niemack, and K. D. Irwin, "Corrugated silicon platelet feed horn array for CMB polarimetry at $150 \mathrm{GHz}$," Society of Photo-Optical Instrumentation Engineers (SPIE) Conference Series, vol.7741, July 2010.

[22] S. R. Golwala, C. Bockstiegel, S. Brugger, N. G. Czakon, P. K. Day, T. P. Downes, R. Duan, J. Gao, A. K. Gill, J. Glenn, M. I. Hollister, H. G. LeDuc, P. R. Maloney, B. A. Mazin, S. G. McHugh, D. Miller, O. Noroozian, H. T. Nguyen, J. Sayers, J. A. Schlaerth, S. Siegel, A. K. Vayonakis, P. R. Wilson, and J. Zmuidzinas, "Status of MUSIC, the MUltiwavelength sub/millimeter inductance camera," Society of Photo-Optical Instrumentation Engineers (SPIE) Confer- ence Series, vol.8452, Sept., 2012.

[23] J. Bland-Hawthorn and P. Kern, "Molding the flow of light: Photonics in astronomy," Phys. Today, vol.65, no.5, pp.31, May 2012.

[24] J. Bland-Hawthorn and P. Kern, "Astrophotonics: a new era for astronomical instruments," Opt. Express, vol.17, no.3, pp.1880-1884, Feb. 2009.

[25] E. Le Coarer, S. Blaize, P. Benech, I. Stefanon, A. Morand, G. Lérondel, G. Leblond, P. Kern, J. M. Fedeli, and P. Royer, "Wavelength-scale stationary-wave integrated Fourier-transform spectrometry," Nat. Photonics, vol.1, no.8, pp.473-478, Aug. 2007.

[26] N. Cvetojevic, N. Jovanovic, C. Betters, J. S. Lawrence, S. C. Ellis, G. Robertson, and J. Bland-Hawthorn, "First starlight spectrum captured using an integrated photonic micro-spectrograph," Astron. Astrophys., vol.544, no.L1, pp.4, Aug. 2012.

[27] M. Mansuripur, "The uncertainty principle in classical optics," Optics \& Photonics News, vol.13, pp.44-48, Jan. 2002.

[28] J. Zmuidzinas, A. Karpov, D. Miller, F. Rice, H. G. Leduc, J. Pearson, and J. A. Stern, "Coherent detection and SIS mixers," Far-IR, Sub-mm and MM Detector Technology Workshop, 2002.

[29] F. Chen, A. J. Sirois, R. W. Simmonds, and A. J. Rimberg, "Introduction of a dc bias into a high- $Q$ superconducting microwave cavity," Appl. Phys. Lett., vol.98, no.13, pp.132509, Mar. 2011.

[30] B. Ho Eom, P. K. Day, H. G. Leduc, and J. Zmuidzinas, "A wideband, low-noise superconducting amplifier with high dynamic range," Nat. Phys., vol.8, no.8, pp.623-627, Aug. 2012.

[31] R. Barends, N. Vercruyssen, A. Endo, P. J. de Visser, T. Zijlstra, T. M. Klapwijk, P. Diener, S. J. C. Yates, and J. J. A. Baselmans, "Minimal resonator loss for circuit quantum electrodynamics," Appl. Phys. Lett., vol.97, no.2, pp.023508, July 2010.

[32] J. Wenner, R. Barends, R. C. Bialczak, Y. Chen, J. Kelly, E. Lucero, M. Mariantoni, A. Megrant, P. J. J. O’Malley, D. Sank, A Vainsencher, H. Wang, T. C. White, Y. Yin, J. Zhao, A. N. Cleland, and J. M. Martinis, "Surface loss simulations of superconducting coplanar waveguide resonators," Appl. Phys. Lett., vol.99, no.11, pp.113513, Sept. 2011.

[33] J. A. Tauber and N. R. Erickson, "A low-cost filterbank spectrometer for submm observations in radio astronomy," Rev. Sci. Instrum. vol.62, no.5, pp.1288, 1991.

[34] E. Shirokoff, P. S. Barry, C. M. Bradford, G. Chattopadhyay, P. Day, S. Doyle, S. Hailey-Dunsheath, M. I. Hollister, A. Kovács, H. G. Leduc, C. M. McKenney, P. Mauskopf, H. T. Nguyen, R. O'Brient, S. Padin, T. J. Reck, L. J. Swenson, C. E. Tucker, and J. Zmuidzinas, "Design and performance of superspec: An on-chip, KID-based, mm-wavelength spectrometer," J. Low Temp. Phys., vol.176, no.56, pp.657-662, Feb. 2014.

[35] A. Endo, C. Sfiligoj, S. J. C. Yates, J. J. A. Baselmans, D. J. Thoen, S. M. H. Javadzadeh, P. P. van der Werf, A. M. Baryshev, and T. M. Klapwijk, "On-chip filter bank spectroscopy at $600-700 \mathrm{GHz}$ using NbTiN superconducting resonators," Appl. Phys. Lett., vol.103, no.3, pp.032601, July 2013.

[36] A. Endo, P. van der Werf, R. M. J. Janssen, P. J. de Visser, T. M. Klapwijk, J. J. A. Baselmans, L. Ferrari, A. M. Baryshev, and S. J. C. Yates, "Design of an integrated filterbank for DESHIMA: On-chip submillimeter imaging spectrograph based on superconducting resonators," J. Low Temp. Phys., vol.167, no.3-4, pp.341-346, May 2012

[37] S. Hemour, F. Podevin, P. Xavier, D. Rauly, P. Benech, and E. le Coarer, "A wideband standing wave-based spectrometer swifts for the 22-GHz water-line detection," Microw. Opt. Technol. Lett. vol.53, no.1, pp.62-65, Nov. 2011.

[38] N. Boudou, A. Monfardini, C. Hoffmann, F. Podevin, P. Xavier, and M. Calvo, "Development of kinetic inductance stationary-wave integrated fourier-transform spectrometry (SWIFTS)," J. Low Temp. Phys., vol.167, no.3-4, pp.386-391, May 2012.

[39] J. Zmuidzinas, "Thermal noise and correlations in photon detection," Appl. Opt., vol.42, no.25, pp.4989-5008, Sept. 2003.

[40] N. Cvetojevic, N. Jovanovic, J. Lawrence, M. Withford, and J. 
Bland-Hawthorn, "Developing arrayed waveguide grating spectrographs for multi-object astronomical spectroscopy," Opt. Express, vol.20, no.3, pp.2062-2072, Jan. 2012.

[41] G. Cataldo, S. H. Moseley, W. Hsieh, W. Huang, T. Stevenson, and E. Wollack, "Micro-spec: an integrated, direct-detection spectrometer for far-infrared and sub-millimeter astronomy," American Astronomical Society Meeting Abstracts, vol.223, pp.454.47, Jan. 2014.

[42] F. Horst, W. M. J. Green, S. Assefa, S. M. Shank, Y. A. Vlasov, and B. J. Offrein, "Cascaded mach-zehnder wavelength filters in silicon photonics for low loss and flat pass-band wdm (de-)multiplexing," Opt. Express, vol.21, no.10, pp.11652-11658, May 2013.

[43] D. Gottesman, T. Jennewein, and S. Croke, "Longer-baseline telescopes using quantum repeaters," Phys. Rev. Lett., vol.109, no.7, pp.070503, Aug. 2012.

[44] A. Kellerer, "Beating the diffraction limit in astronomy via quantum cloning," Astron. Astrophys., vol.561, pp.A118, Jan. 2014.

[45] J. Clarke and F. K. Wilhelm, "Superconducting quantum bits," Nature News, vol.453, no.7198, pp.1031-1042, June 2008.

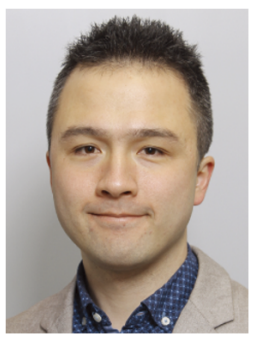

Akira Endo received the Ph.D. degree in astronomy (with "Prize for Encouragement in Research," analogous to cum laude) from the School of Science, University of Tokyo in 2009 with a dissertation entitled "AlN Tunnel Barriers for Submillimeter Wave SIS Mixers." Since 2009, he has been working as a postdoc researcher at the Kavli Institute of Nanoscience within the Delft University of Technology in the Netherlands. From November 2014, he is appointed as a tenure-track assistant professor at the Department of Electrical Engineering, Faculty of Mathematics and Computer Science (EEMCS), at Delft University of Technology. During 2011-2014, he conducted a combined NWO-Veni program and a JSPS postdoctoral research program "Probing the Cosmic History of Star Formation by Submillimeter Wave Ultrabroadband Spectroscopy using Superconducting Resonators." Endo is the co-PI of an NWO-M program "DESHIMA: On-chip Filterbank Spectrometer for Submillimeter Wave Cosmology," and he has recently been granted a NWO-Vidi program starting from 2014 entitled "Dawn of Superconducting Astrophotonics." These projects together aim at the development and demonstration of a superconducting on-chip filterbank spectrometer DESHIMA, which capitalizes on the collaborative development of MKIDs at Delft University of Technology and SRON Netherlands Institute for Space Research. 